\title{
Automated Detection of Pancreatic Cystic Lesions on CT Using Deep Learning
}

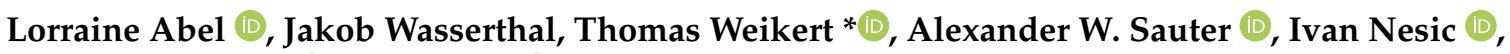 \\ Marko Obradovic $\mathbb{C}^{(0)}$, Shan Yang $\mathbb{C}^{\circ}$, Sebastian Manneck, Carl Glessgen, Johanna M. Ospel, Bram Stieltjes, \\ Daniel T. Boll and Björn Friebe
}

check for updates

Citation: Abel, L.; Wasserthal, J.; Weikert, T.; Sauter, A.W.; Nesic, I.; Obradovic, M.; Yang, S.; Manneck, S.; Glessgen, C.; Ospel, J.M.; et al. Automated Detection of Pancreatic Cystic Lesions on CT Using Deep Learning. Diagnostics 2021, 11, 901. https://doi.org/10.3390/diagnostics 11050901

Academic Editor: Jens Höppner

Received: 30 March 2021

Accepted: 14 May 2021

Published: 19 May 2021

Publisher's Note: MDPI stays neutral with regard to jurisdictional claims in published maps and institutional affiliations.
Clinic of Radiology \& Nuclear Medicine, University Hospital Basel, University of Basel, Petersgraben 4, 4031 Basel, Switzerland; lorraine.abel@usb.ch (L.A.); jakob.wasserthal@usb.ch (J.W.); alexander.sauter@usb.ch (A.W.S.); ivan.nesic@usb.ch (I.N.); marko.obradovic@usb.ch (M.O.); shan.yang@usb.ch (S.Y.); sebastian.manneck@usb.ch (S.M.); carlguillaume.glessgen@usb.ch (C.G.); johanna.ospel@usb.ch (J.M.O.); bram.stieltjes@usb.ch (B.S.); daniel.boll@usb.ch (D.T.B.); bjoern.friebe@usb.ch (B.F.)

* Correspondence: thomas.weikert@usb.ch

\begin{abstract}
Pancreatic cystic lesions (PCL) are a frequent and underreported incidental finding on CT scans and can transform into neoplasms with devastating consequences. We developed and evaluated an algorithm based on a two-step nnU-Net architecture for automated detection of PCL on CTs. A total of 543 cysts on 221 abdominal CTs were manually segmented in 3D by a radiology resident in consensus with a board-certified radiologist specialized in abdominal radiology. This information was used to train a two-step nnU-Net for detection with the performance assessed depending on lesions' volume and location in comparison to three human readers of varying experience. Mean sensitivity was $78.8 \pm 0.1 \%$. The sensitivity was highest for large lesions with $87.8 \%$ for cysts $\geq 220 \mathrm{~mm}^{3}$ and for lesions in the distal pancreas with up to $96.2 \%$. The number of false-positive detections for cysts $\geq 220 \mathrm{~mm}^{3}$ was 0.1 per case. The algorithm's performance was comparable to human readers. To conclude, automated detection of PCL on CTs is feasible. The proposed model could serve radiologists as a second reading tool. All imaging data and code used in this study are freely available online.
\end{abstract}

Keywords: pancreatic cystic lesion; intraductal papillary mucinous neoplasia; tomography; X-ray computed; detection; artificial intelligence; deep learning; nnU-Net

\section{Introduction}

Pancreatic cystic lesions (PCLs) are a common finding in cross-sectional imaging. The prevalence of incidental pancreatic cysts in abdominal CTs range from 2.6 to $5.4 \%$ in the normal population [1,2], increasing with age [1,3]. On an MRI, this prevalence is even higher with up to $45 \%$ [4].

Whereas non-neoplastic lesions, like retention cysts or pseudocysts consecutive to pancreatitis, are not at risk for malignant transformation, mucinous cysts are considered potential cancer precursors [5]. Around 90\% of non-inflammatory PCLs are intraductal papillary mucinous neoplasms (IPMNs), mucinous cystic neoplasms and serous cystadenomas [3]. In surgical studies, IPMNs represent the most frequent premalignant PCLs and about $50 \%$ of all resected pancreatic cysts [6,7]. They are topographically classified according to their relation with the main pancreatic duct (MPD), with progression to invasive cancer in $42-48 \%$ of the main duct-IPMNs and mixed type-IPMNs, and $11-26 \%$ of the BD-IPMNs $[5,6,8,9]$. Apart from the malignant degeneration of an IPMN into an invasive carcinoma, the rate of concomitant carcinoma in the presence of an IPMN can be as high as $4.4 \%$ to $11.2 \%$ of patients [10-12]. 
The performance of radiologists in making precise and consistent diagnoses is challenged by the increasing workload and associated fatigue [13,14]. In a large study involving radiology reports written by residents, Vosshenrich et al. found higher rates of incongruences in conjunction with increasing work hours [13]. Surrogate endpoints for physicians ${ }^{\prime}$ fatigue, like detection of pathology and diagnosis accuracy [15], could benefit from the help of artificial intelligence (AI) [16,17]. Over the past few decades, several AI-algorithms have proven their performance in radiology [18-22], reducing the number of missed findings and false-positive findings (FPs) [23]. Furthermore, automated pathology detection allows radiologists to put their capacities into more complex tasks, such as making the final diagnosis [24,25].

Automated detection of precursor lesions for pancreatic cancer, which PCLs are a part of, would help with appropriate surveillance. This task is technically challenging. The pancreas and its cysts represent a very small part of the entire pool of voxels of a CT scan, often only about $1 \%$ and $0.1 \%$, respectively [26]. The current state of the art for the automatic segmentation of the pancreas uses organ-attention networks with reverse connections to achieve a mean Dice-Sørensen coefficient (DSC) of $87.8 \pm 3.1 \%$ [27]. The number of algorithms for the challenging detection of pancreatic cysts are very limited and none are clinically implemented [26,28].

The aim of this study is to develop and test an nnU-Net algorithm for automated detection of pancreatic cystic lesions. It could help radiologists to cope with increasing numbers of imaging tests and reduce the numbers of PCLs not mentioned in radiology reports, therefore potentially improving early diagnosis of pancreatic cancer.

\section{Materials and Methods}

This retrospective study was approved by the local Institutional Review Board (Ethikkommission Nordwest- und Zentralschweiz; project-ID: Req-2021-00216). Patient data was fully anonymized.

\subsection{Data Selection}

All consecutive abdominal CTs acquired at our institution between January 2010 and October 2020, and meeting the criteria mentioned below were identified with an in-house developed RIS/PACS search engine [29]. In order to identify all relevant cases despite the plethora of terms describing PCLs in radiology reports, we used multiple search strings, which are documented in Appendix A.

The inclusion criteria were: (I) CT scan of the abdomen in portal venous phase; (II) slice thickness of 1-1.5 mm; and a (III) formal description of a PCL in the written radiology report. The exclusion criteria were: (I) disagreement of patient to use their data; (II) formal report describing a pancreatic tumor; (III) patient with acute or chronic pancreatitis based on clinical history or report; (IV) images with movement or beam hardening artefacts described in the report; and (V) pseudocysts. If there were more than one study of a patient, only the most recent $\mathrm{CT}$ was selected.

For the resulting 221 studies, a curated dataset based on the radiology reports was compiled, documenting the location (uncinate process, head, body, tail) and size (mm) of each cyst, if specified. Whenever provided, we retrieved the diagnosis suggested as most probable for the PCLs from the reports. Patient characteristics at the time of CT acquisition were collected from the clinical information system. Figure 1 shows the detailed data selection flowchart. 


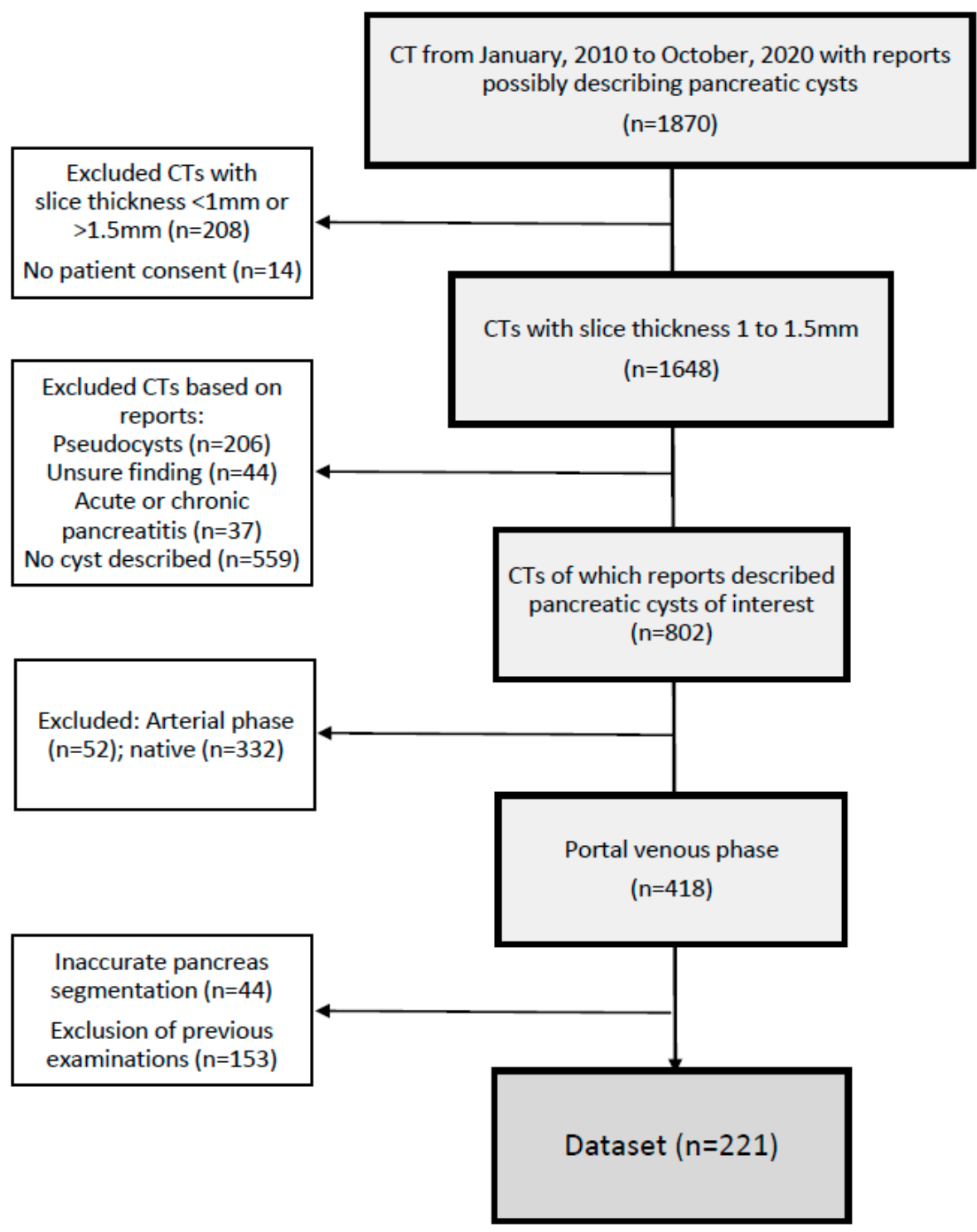

Figure 1. Study selection flowchart. The starting point was the collection of abdominal CTs with reports possibly describing PCL based on the search strings documented in Appendix A. At a later selection stage, based on comprehensive assessment of each report by a radiology resident, reports that did not describe PCLs or described other findings like signs of acute or chronic pancreatitis, were excluded.

\subsection{Patient Characteristics and Radiology Report Information}

The final dataset comprised 221 series matching our inclusion criteria. Patients' mean age was $72.9 \pm 12.7$ years and 138 were female $(62.4 \%)$. The information related to the cysts described in radiology reports is summarized in Table 1. An assumption on the most probable diagnosis was missing in 36 reports. 
Table 1. Cyst size and most probable diagnosis as provided in the 221 radiology reports. Information on the exact number of PCLs and their size was provided in 199 and 194 reports, respectively. Minimal and maximal diameter represent the measurements of the cysts given on reports. An average diameter was calculated only if both were mentioned.

\begin{tabular}{|c|c|c|c|}
\hline Parameter & $n(\%)$ & $\begin{array}{c}\text { Mean }\left( \pm S^{1}\right) \\
\text { in } \mathbf{~ m m}\end{array}$ & $\begin{array}{c}\text { Median } \\
\text { in } \mathrm{mm}\end{array}$ \\
\hline Reported number of cysts per patient & & $1.2(0.4)$ & \\
\hline \multicolumn{4}{|l|}{ Size: } \\
\hline Maximal diameter & & $12.8(7.7)$ & 12.0 \\
\hline Minimal diameter & & $11.6(7.3)$ & 10.0 \\
\hline Mean diameter & & $13.3(7.4)$ & 11.5 \\
\hline \multicolumn{4}{|l|}{ Radiologically suspected diagnosis: } \\
\hline IPMN & $173(78.3)$ & & \\
\hline Indeterminate & $36(16.2)$ & & \\
\hline $\mathrm{SCN}^{2}$ & $5(2.3)$ & & \\
\hline $\mathrm{MCN}^{3}$ & $5(2.3)$ & & \\
\hline Others (lymphangioma, ontogenetic cyst) & $2(0.9)$ & & \\
\hline
\end{tabular}

${ }^{1}$ standard deviation; ${ }^{2}$ serous cystic neoplasm; ${ }^{3}$ mucinous cystic neoplasm.

\subsection{CT Protocols}

CT examinations were performed on the following scanners: SOMATOM Definition Edge $(n=68)$, SOMATOM Definition AS+ $(n=52)$, SOMATOM Definition Flash $(n=86)$, SOMATOM Force $(n=3)$, Emotion $16(n=11)$ (all Siemens Healthcare), and GE LightSpeed VCT $(n=1)$ (GE Healthcare). Slice thickness was $1.49 \pm 0.1 \mathrm{~mm}$. Mean tube current was $327.9 \pm 133.4 \mathrm{mAs}$ and mean peak kilovoltage was $109.1 \pm 9.9 \mathrm{kVp}$. Contrast agent was administered with injection rates ranging from 1.5 to $3.5 \mathrm{~mL} / \mathrm{s}$, using Ultravist or Iopamiro (both $370 \mathrm{mg}$ iodine per $\mathrm{mL}$ ).

\subsection{Data Preprocessing: Cropping of CTs to the Region Showing the Pancreas}

Based on the fact that PCLs are anatomically strictly associated with the pancreas, the first step was automatic segmentation of the organ using a nnU-Net pretrained on the 282 CTs of the pancreas (portal venous phase) from the public Medical Segmentation Decathlon, reaching a DSC of $82 \%$ [30]. Based on the predicted segmentations of the pancreas, the abdominal CT scans were cropped to the CT slices that show the organ. All cropped CTs were reviewed by the main reader slice-by-slice (L.A.) and excluded in the case of incomplete pancreas segmentation.

\subsection{Ground-Truth Generation}

\subsubsection{Segmentation of PCLs}

The medical image editing software NORA (University of Freiburg, Freiburg, Germany) was used by a supervised radiology resident in their first year of professional education (L.A.) to perform fully manual 3D-segmentation of all PCLs, reaching a subjective accuracy of about two voxels at cyst margin [31]. Subsequently, all segmentations were reviewed by a board-certified radiologist with 11 years of experience in abdominal radiology (B.F.) who could overrule the decisions of the first reader.

This resulted in 543 manually segmented cysts that constituted the ground truth (GT), with $2.5 \pm 2.0$ cyst per case on average. Volumes ranged between $10.2 \mathrm{~mm}^{3}$ and $39,973.5 \mathrm{~mm}^{3}$, with a mean of $1004.9 \mathrm{~mm}^{3}$.

\subsubsection{Segmentation of Main Pancreatic Ducts (MPD)}

MPDs potentially resemble a PCL, which might mislead the model. To overcome this problem, we additionally provided the model with a manual segmentation of the MPD in all 221 subjects as a separate class. Accessory ducts were not segmented. 


\subsection{Algorithm}

For the detection of PCLs, we trained a nnU-Net on our manually annotated dataset [30]. NnU-Nets are a medical segmentation framework, which automatically configures the data preprocessing as well as the hyperparameters for training a U-Net. They are able to derive heuristics for optimally setting the data preprocessing parameters (e.g., normalization and resampling) as well as the U-Net configuration (e.g., number of layers and batch size) based on the characteristics of the input dataset. Furthermore, they perform extensive data augmentations (image rotation, blurring, etc.). On more than 20 public imaging segmentation challenges, this automatically configured segmentation pipeline was superior to other submissions. For this reason, we chose to use the nnU-Net for our project.

For our purposes, we were interested in finding PCLs (=detection) and not their precise outline (=segmentation). We used segmentation maps returned by the nnU-Net for PCL detection by using connected component analysis to convert the binary cyst segmentation into a cyst instance segmentation. A lesion was considered detected if the predicted segmentation overlapped at least 30\% (in terms of DSC) with the GT segmentation. Besides PCLs, the algorithm was trained to detect MDPs as a second class to improve PCL detection. As PCLs were at the focus of this study, MDP segmentations were not analyzed in detail. Processing times were recorded. The framework of our approach is shown in Figure 2.

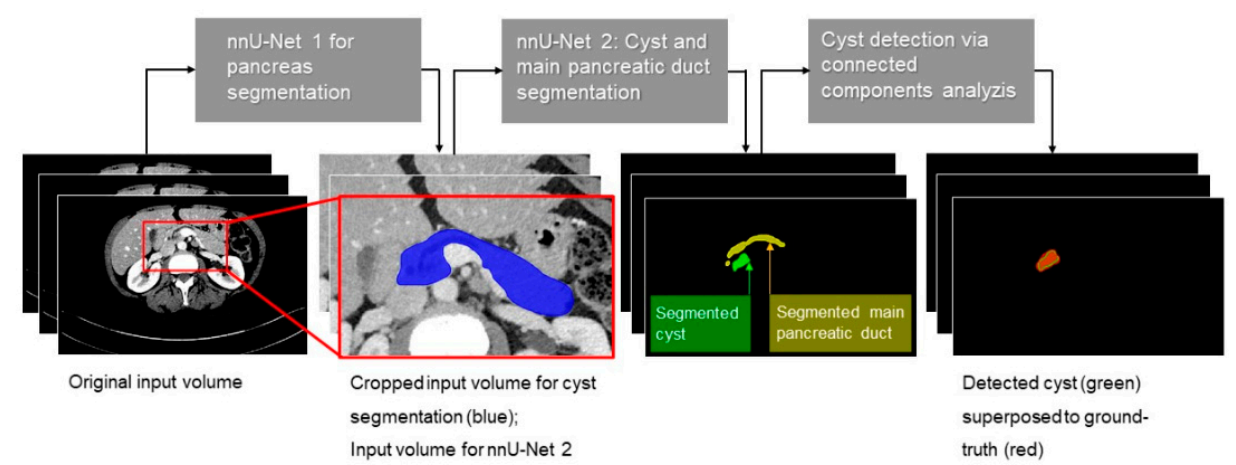

Figure 2. Structure of our segmentation approach. First, the original input volume was cropped based on the automatic segmentation of the pancreas with a first nnU-Net. PCLs and MPDs were then manually segmented on the cropped volume to create a ground truth. The trained algorithm detected cysts. The image on the right shows the resulting cyst detection superposed to the ground truth.

For evaluation of the model, five-fold cross-validation was used to include each sample in the testing set once. This is statistically sound because, in nnU-Nets, the hyperparameters are chosen by fixed heuristics prior to training. We excluded PCLs with a volume below $10 \mathrm{~mm}^{3}$, considering the difficulty of their segmentation, even for humans, and their low clinical relevance [32].

\subsection{Performance Subanalyses Regarding PCL Size and Location within the Pancreas}

Apart from general performance measures, the performance for different sizes of PCLs was analyzed. Furthermore, in order to assess the difference in performance according to the main regions of the pancreas, we automatically split the pancreatic parenchyma into three equal volumes along the centerline of the pancreas mask. The proximal third roughly corresponds to both the head and uncinate process. The middle third corresponds to the body, and the distal third to the tail. PCLs located in two regions were attributed to the region in which most voxels of the ground truth mask were located.

\subsection{Comparison of Model's Performance with Human Readers}

Using the medical imaging platform NORA, one reader with seven (S.M.) and two readers with four years of experience in diagnostic radiology (J.O., C.G.) manually annotated the linear diameter (in $\mathrm{mm}$ ) of each PCL on the orientation where it was the greatest (axial, coronal or sagittal) on 47 randomly selected, cropped pancreatic series from the 
training dataset [33]. A cyst was considered successfully detected by the rater if a 3D-sphere drawn around its linear diameter overlapped with the GT-segmentation by at least 10\% (in terms of DSC). Each rater was compared to the GT in terms of sensitivity and FPs.

\subsection{Statistical Analysis}

Statistical analysis was performed with SPSS Statistics, version 25 (IBM Corp., Armonk, NY, USA). We assessed the detection rate of the model according to the cyst location in either of the three regions and to their volume group with the chi-square test. A paired sample t-test was used for comparing predictions and GT regarding PCL volumes and of the mean number of lesions per patient. A McNemar test was used to compare dichotomous traits between GT and predictions. $p$-values $<0.05$ were considered statistically significant.

\section{Results}

\subsection{General Performance}

The fully automated detection model took $1 \mathrm{~min} 43 \mathrm{sec}$ on average, on a modern computer with an NVIDIA GPU, to automatically detect PCLs in abdominal CT scans. The mean sensitivity for all cases was $78.8 \pm 0.1 \%$. There were 0.48 FPs per case. The difference in lesions count per patient between GT (2.47 in average) and predictions (1.76 in average) was significant $(p<0.001)$. In total, 5 of 44 false-positive findings were caused by MPDs (11.4\%). Figure 3 provides examples of correct PCL detection, false-positive and false-negative findings.

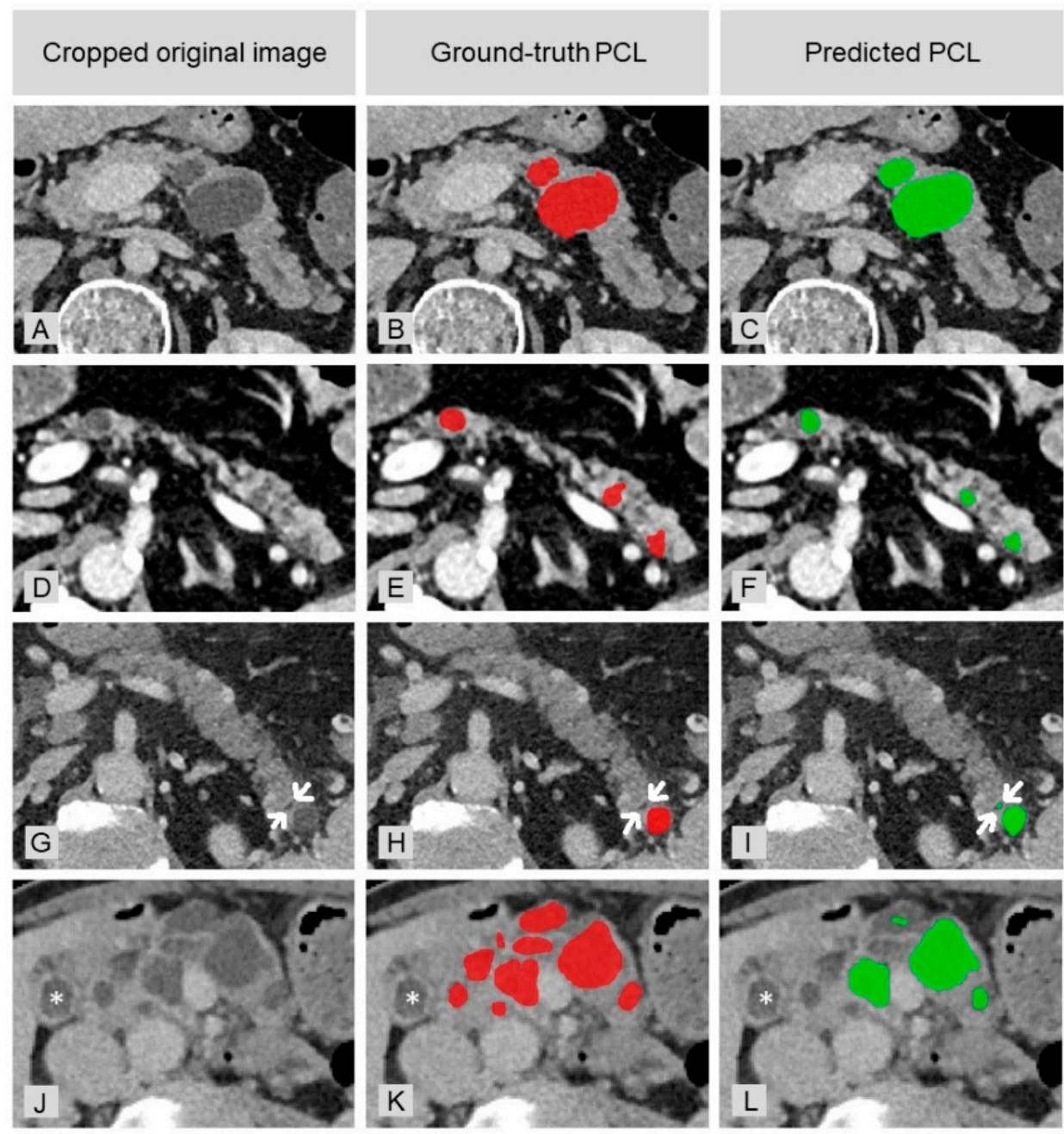

Figure 3. Examples of detected PCLs and manually segmented cysts of the GT in comparison with the original images: (A-C) true positive detection of large cysts; (D-F) true positive detection of smaller cysts; (G-I) false-positive finding (small fat interdigitation; marked with arrows); and ( $\mathbf{J}-\mathbf{L})$ failure at detecting multiple cysts. The star indicates the gallbladder. 


\subsection{Performance Sub-Analyses}

\subsubsection{Performance Depending on Cyst Volume}

PCLs were assigned to four groups based on their volume to assess the impact of different volumes on the model's performance. Table 2 provides information on the performance of the model within distinct volume groups. Sensitivity markedly increased with PCL volume to up to $91.9 \%$ for volumes $\geq 600 \mathrm{~mm}^{3}$. In parallel, FPs were rarest for these volumes, with 0.08 per case. Figure 4 shows the sensitivity and frequency of FPs as a function of PCL volumes.

Table 2. Sensitivity, FPs/Case and F1-score of the model regarding detection of PCLs as a function of volume groups of PCLs.

\begin{tabular}{cccc}
\hline Cyst Volume Group $\left[\mathrm{mm}^{\mathbf{3}}\right]$ & Sensitivity $\mathbf{( \% )}$ & FPs/Case & F1-Score \\
\hline $10-50$ & 40.1 & 0.33 & 0.40 \\
$>50-200$ & 65.5 & 0.19 & 0.66 \\
$>200-600$ & 75.9 & 0.11 & 0.76 \\
$>600$ & 91.9 & 0.08 & 0.91 \\
\hline
\end{tabular}

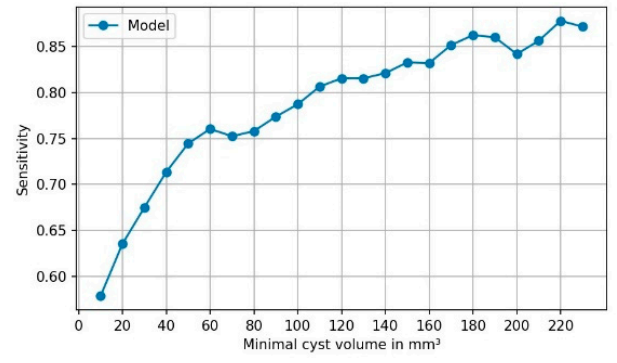

(a)

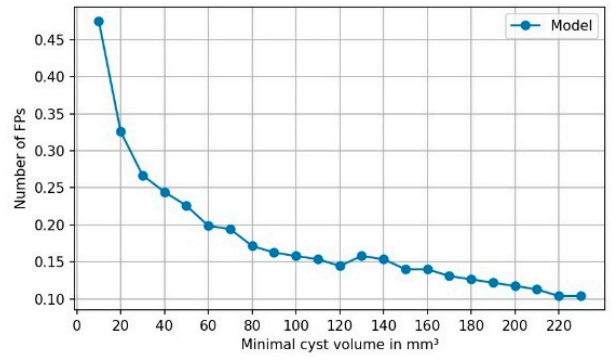

(b)

Figure 4. The (a) sensitivity and (b) FP rate of model's predictions as a function of cyst volume. Each dot indicates the sensitivity and FP rate for cysts of equal or larger volume.

\subsubsection{Performance Depending on Cysts' Location within Pancreas}

The number of PCLs present in the GT did not significantly differ from the number of PCLs predicted by the model within each fictive pancreas region and neither did the detection rate significantly differ between regions $(p=0.379)$. Figure 5 shows the performance of the model in the three regions of the pancreas. Sensitivity was highest and the number of FPs per case was lowest in the distal part of the pancreas.

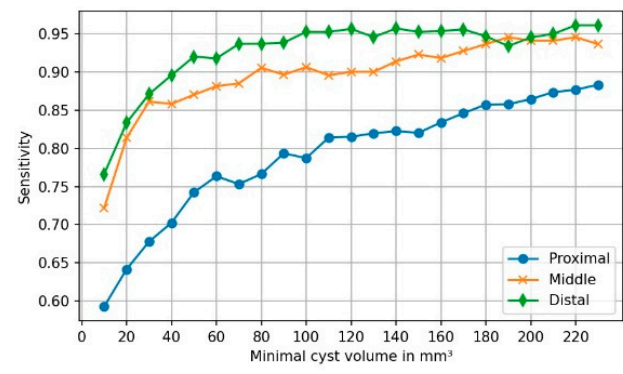

(a)

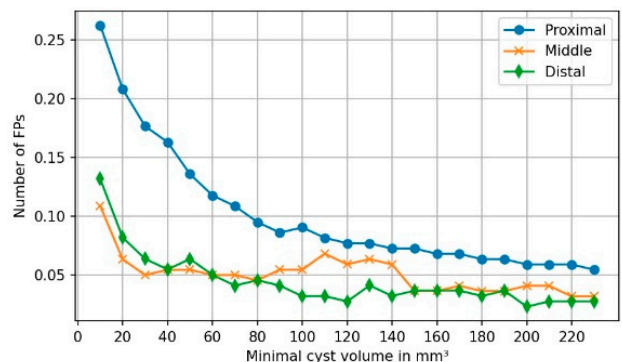

(b)

Figure 5. (a) Sensitivity and (b) false positive (FP) rate of model's predictions as a function of cyst volume, for each region of the pancreas.

\subsection{Comparison of Model's Performance with Human Readers}

Figure 6 compares the sensitivity of the three readers and the model. The model moderately outperformed the readers for all cyst volumes. However, for very small 
PCLs $\leq 40 \mathrm{~mm}^{3}$, two readers defined less FPs than the model. This trend inverses for volumes $\geq 200 \mathrm{~mm}^{3}$, with the model finding approx. 0.2 less false-positive PCLs per case than the most experienced reader.

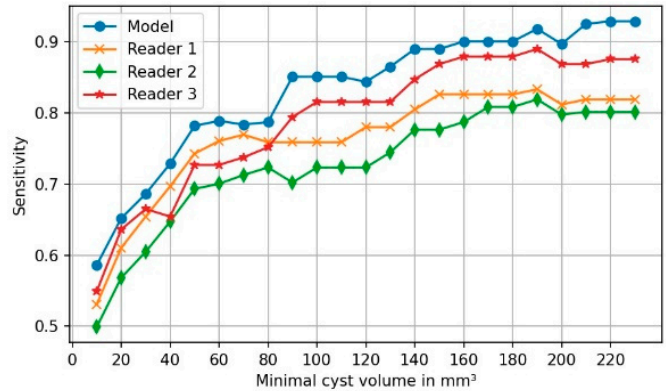

(a)

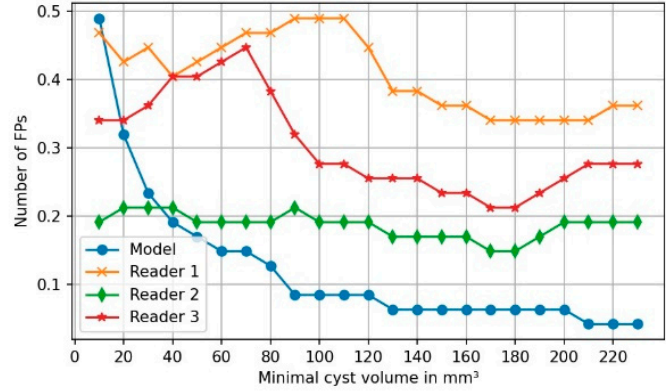

(b)

Figure 6. Comparison of the model with three human readers for (a) detection rates of PCL and (b) false-positive findings per case, as a function of cysts' volumes. Of note, this analysis is based on a subset of 47 CTs.

Compared to human readers, our model performed best in terms of FPs in the proximal part of the pancreas, especially for PCLs $\geq 60 \mathrm{~mm}^{3}$. Detailed results are provided as graphs in Appendix B.

\section{Discussion}

The aim of this study was to develop and evaluate an algorithm for automatic detection of pancreatic cystic lesions. While AI has demonstrated excellent performance for segmentation of organs with sharp borders like the lungs [34], organs with fuzzy delineation like the pancreas (e.g., caused by fat interdigitations) and detection of lesions within these entities remain a challenging task for algorithms [35]. The overall sensitivity of the algorithm in detecting PCLs on abdominal CTs was 78.8\%. Sensitivity increased with the volume of the lesions up to $87.8 \%$ on average for lesions $>220 \mathrm{~mm}^{3}$. This is expected, as more voxels per lesion make it easier for the model to detect a PCL amongst the entire voxels of an abdominal CT. The weaker performance of our model on smaller cysts has to be put into perspective with their low clinical relevance. The American College of Radiology showed the absence of growth on the 3 year follow-up of PCLs smaller than $5 \mathrm{~mm}$ at detection [32,36]. Overall, the presence of a PCL of $30 \mathrm{~mm}$ or larger is considered an independent risk factor for malignancy $[4,37]$. Of note, the rate of FPs decreased to a minimum of 0.1 per case with increasing cyst volumes.

Sensitivity was highest in the distal pancreas, reaching up to $96.2 \%$. This can be partly explained by the fact that the proximal pancreas is in close anatomical relation to structures which resemble PCLs on cross-sectional imaging (e.g., bile duct, choledochal cyst, and duodenal diverticula), which can confuse the model. Nonetheless, the model showed high detection rates in the proximal region of the organ as well, corresponding to the head and uncinate process, where two other groups found the IPMN to be the most frequent $[8,9]$. Regarding the comparison to human readers, the algorithm showed moderately superior detection rates compared to three radiology residents, and detected less FPs. Therefore, it could be used as a second reading tool for helping radiologists not to miss PCLs. The actual benefit of this application in clinical routine and against the backdrop of current guidelines remains to be evaluated in future research $[4,33,38,39]$.

In their attempt to create an algorithm for automated PCL segmentation, Zhou et al. trained their initial model on their own dataset of 131 cystic pancreas segmentations, achieving a DSC of $63.44 \pm 27.71 \%$ for cyst segmentation at testing in 2017 [26], and $68.98 \pm 26.68 \%$ with their most recent algorithm [28]. As the aim of the study at hand was PCL detection, the results cannot be directly compared. 
We chose the task of PCL detection on CT, considering its good spatial resolution, lower sensitivity to motion artifacts, and the fact that abdominal CT is frequently performed. Thin section, high-resolution, contrast-enhanced CT was found to provide enough details regarding the structure of PCLs to make a diagnosis [40] and Lee, J. et al. recently declared MRI and CT to be interchangeable for assessment and follow-up of patients with PCLs [41]. For patients refusing an MRI, pancreatic $\mathrm{CT}$ is the recommended alternative modality according to the societies ICG, ACG and ESG [32], and offers a comparable accuracy to MRCP in terms of PCL characterization [42]. However, we recognize the high diagnostic value of other modalities such as multi-parametric MRI, MRCP, and endoscopic ultrasound (EUS) $[4,32,43,44]$. Consequently, even though our model provides help for the detection of PCLs on CT, models for other modalities would be useful for their accurate characterization.

This study has limitations. First, the PCL detection algorithm was trained and tested on data from a single medical center, which limited the amount of available data. Second, due to the lack of external validation, we cannot make a clear statement on the performance of our algorithm on external data. However, given the high degree of standardization of CT protocols, we do not expect a major drop in performance. Third, main duct-IPMN were not included in the training as the sample size was too small and their morphologic presentation is too different from other PCLs. Fourth, the first step (segmentation of the pancreas) failed in 44 cases, which has a negative impact on direct clinical implementability. Possible remedies are to improve the organ segmentation algorithm using more training data or adding a verification step of the first algorithm's output ("whole pancreas included in the scan or not?"), e.g., by a third algorithm. Having exclusively confronted our model with a pre-screened set of examinations containing PCLs and excluding pancreatitis and tumorous pancreas, the amount of FPs might increase when used in a clinical setting, which constitutes the fifth limitation of this study.

\section{Conclusions}

This study shows that automated AI-based detection of pancreatic cystic lesions on contrast-enhanced CT is possible with good diagnostic performance comparable to that of radiologists. As it has become clearer that a relevant proportion of pancreatic cysts, which are an underreported entity on CTs, will develop into malignant lesions, the diagnosis and surveillance of pancreatic cysts is gaining importance. The algorithm presented in this study could help to improve the accuracy of detection and surveillance of PCLs by serving radiologists as a second reading tool.

Author Contributions: Conceptualization, B.F., A.W.S., and T.W.; methodology, B.F., L.A., A.W.S., T.W. and J.W.; software, J.W., I.N., M.O. and S.Y.; validation, J.W., L.A., J.W., A.W.S. and B.F.; formal analysis, L.A. and J.W.; investigation, L.A., J.W., S.Y., S.M., C.G. and J.M.O.; resources, L.A. and S.Y.; data curation, J.W.; writing—original draft preparation, L.A.; writing—review and editing, L.A., T.W., J.W., I.N., M.O. and A.W.S.; visualization, L.A., J.W. and T.W.; supervision, B.F., T.W., D.T.B. and B.S.; project administration, B.F., L.A., A.W.S. and T.W. All authors have read and agreed to the published version of the manuscript.

Funding: This research received no external funding.

Institutional Review Board Statement: The study was conducted according to the guidelines of the Declaration of Helsinki and approved by the local Institutional Review Board (Ethikkommission Nordwest- und Zentralschweiz; project-ID: Req-2021-00216; Date of approval: 3 March 2021).

Informed Consent Statement: Informed consent was obtained from all subjects involved in the study.

Data Availability Statement: All data and algorithms used in this study are openly available at Zenodo: anonymized CT image dataset of 221 patients, manual segmentations of all pancreatic cysts and main pancreatic ducts, and the code of both deep learning algorithms (nnU-Nets). To access, please follow this link: 10.5281 /zenodo.4621056.

Acknowledgments: We thank Christophe Aberle, University Hospital Basel, Switzerland, for assisting with the data collection. 
Conflicts of Interest: The authors declare no conflict of interest.

\section{Appendix A}

PACS search queries, English translation in brackets.

*Pseudozyste* (pseudocyst) OR *IPMN* OR *pankreasschwanzzyste* (cyst of pancreatic tail) OR * pankreaskopfzyste* (cyst of head of pancreas) OR *pankreaszyste* (pancreatic cyst) OR "uncinatus zyste" (cyst of uncinate process) 5 OR "pankreasschwanz zystisch" (cystic pancreatic tail) $~ 5$ OR "cauda zyste" (cyst of cauda) 5 OR "Schwanz zyste" (cyst of tail) 5 OR "pankreasschwanz zystisch" (cystic pancreatic tail) 5 OR "Pankreasschwanz zyste" (cyst of pancreatic tail) 5 OR "Pankreascorpus zyste" (cyst of pancreatic corpus) 5 OR "corpus zyste" $\sim 5$ (cyst of corpus) OR " $\sim 5$ OR "caput zyste" (cyst of caput) $\sim 5$ OR "kopf zyste" (head cyst) 5 OR "pankreaskopf zystisch" (cystic head of pancreas) 5 OR "pankreaskopf zyste" (cyst of head of pancreas) 5 OR "pankreasparenchym zystisch" (cystic pancreatic parenchyma) 5 OR "pankreasparenchym zyste" (cyst of pancreatic parenchyma) 5 OR "pankreas zystisch" (cystic pancreas) 5 OR "bauchspeicheldrüse zyste" (cystic pancreas) 5 OR "pankreas zyste" (pancreatic cyst) $\sim 5$ OR "pancreas zyste" (pancreatic cyst) 5

\section{Appendix B}
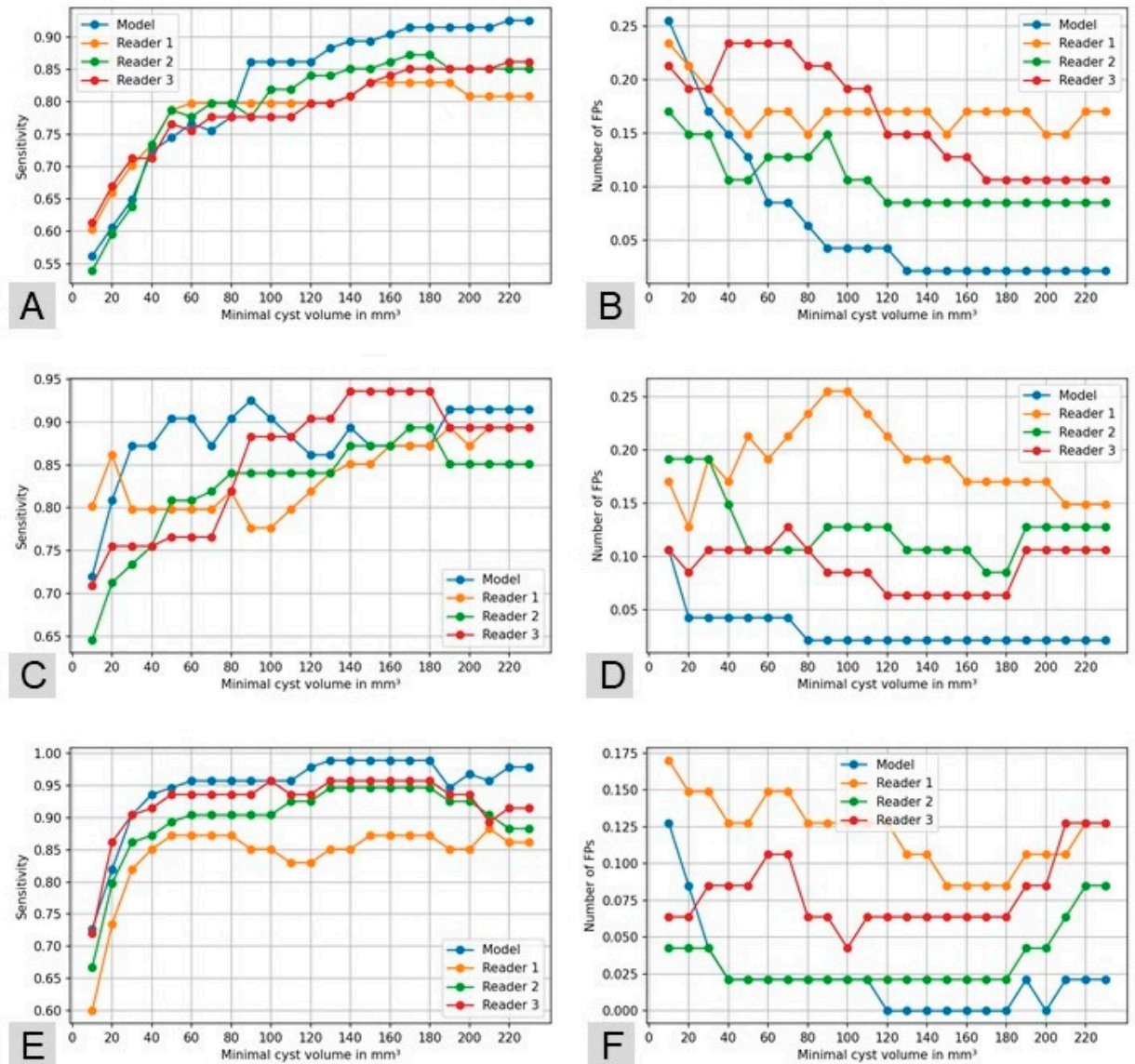

Figure A1. Comparison of each reader with the model's predictions for each of the three pancreas regions: (A,B) proximal; (C,D) middle; (E,F) distal. 


\section{References}

1. $\quad$ Laffan, T.A.; Horton, K.M.; Klein, A.P.; Berlanstein, B.; Siegelman, S.S.; Kawamoto, S.; Johnson, P.T.; Fishman, E.K.; Hruban, R.H. Prevalence of Unsuspected Pancreatic Cysts on MDCT. AJR Am. J. Roentgenol. 2008, 191, 802-807. [CrossRef]

2. Zanini, N.; Giordano, M.; Smerieri, E.; Cipolla d'Abruzzo, G.; Guidi, M.; Pazzaglini, G.; De Luca, F.; Chiaruzzi, G.; Vitullo, G.; Piva, P.; et al. Estimation of the Prevalence of Asymptomatic Pancreatic Cysts in the Population of San Marino. Pancreatology 2015, 15, 417-422. [CrossRef]

3. Kromrey, M.-L.; Bülow, R.; Hübner, J.; Paperlein, C.; Lerch, M.M.; Ittermann, T.; Völzke, H.; Mayerle, J.; Kühn, J.-P. Prospective Study on the Incidence, Prevalence and 5-Year Pancreatic-Related Mortality of Pancreatic Cysts in a Population-Based Study. Gut 2018, 67, 138-145. [CrossRef]

4. European Evidence-Based Guidelines on Pancreatic Cystic Neoplasms. Gut 2018, 67, 789-804. [CrossRef]

5. Chakraborty, J.; Midya, A.; Gazit, L.; Attiyeh, M.; Langdon-Embry, L.; Allen, P.J.; Do, R.K.G.; Simpson, A.L. CT Radiomics to Predict High-Risk Intraductal Papillary Mucinous Neoplasms of the Pancreas. Med. Phys. 2018, 45, 5019-5029. [CrossRef]

6. Barkin, J.A.; Barkin, J.S. Pancreatic Cysts: Controversies, Advances, Diagnoses, and Therapies. Pancreas 2017, 46, 735-741. [CrossRef] [PubMed]

7. Ferrone, C.R.; Correa-Gallego, C.; Warshaw, A.L.; Brugge, W.R.; Forcione, D.G.; Thayer, S.P.; Castillo, C.F. Current Trends in Pancreatic Cystic Neoplasms. Arch. Surg. Chic. Ill 1960 2009, 144, 448-454. [CrossRef] [PubMed]

8. Crippa, S.; Castillo, C.F.; Salivia, R.; Finkelstein, D.; BASSI, C.; Dominguez, I.; Muzikansky, A.; Thayer, S.P.; Falconi, M.; MinoKenudson, M.; et al. Mucin-Producing Neoplasms of the Pancreas: An Analysis of Distinguishing Clinical and Epidemiologic Characteristics. Clin. Gastroenterol. Hepatol. Off. Clin. Pract. J. Am. Gastroenterol. Assoc. 2010, 8, 213-219. [CrossRef] [PubMed]

9. Rodriguez, J.R.; Salivia, R.; Crippa, S.; Warshaw, A.L.; Bassi, C.; Falconi, M.; Thayer, S.P.; Lauwers, G.Y.; Capelli, P.; MinoKenudson, M.; et al. Branch-Duct Intraductal Papillary Mucinous Neoplasms: Observations in 145 Patients Who Underwent Resection. Gastroenterology 2007, 133, 72-310. [CrossRef] [PubMed]

10. Kanno, A.; Satoh, K.; Hirota, M.; Hamada, S.; Umino, J.; Itoh, H.; Masamune, A.; Asakura, T.; Shimosegawa, T. Prediction of Invasive Carcinoma in Branch Type Intraductal Papillary Mucinous Neoplasms of the Pancreas. J. Gastroenterol. 2010, 45, 952-959. [CrossRef]

11. Sahora, K.; Crippa, S.; Zamboni, G.; Ferrone, C.; Warshaw, A.L.; Lillemoe, K.; Mino-Kenudson, M.; Falconi, M.; Fernandez-del Castillo, C. Intraductal Papillary Mucinous Neoplasms of the Pancreas with Concurrent Pancreatic and Periampullary Neoplasms. Eur. J. Surg. Oncol. EJSO 2016, 42, 197-204. [CrossRef] [PubMed]

12. Ideno, N.; Ohtsuka, T.; Kono, H.; Fujiwara, K.; Oda, Y.; Aishima, S.; Ito, T.; Ishigami, K.; Tokunaga, S.; Ohuchida, K.; et al. Intraductal Papillary Mucinous Neoplasms of the Pancreas With Distinct Pancreatic Ductal Adenocarcinomas Are Frequently of Gastric Subtype. Ann. Surg. 2013, 258, 141-151. [CrossRef] [PubMed]

13. Vosshenrich, J.; Brantner, P.; Cyriac, J.; Boll, D.T.; Merkle, E.M.; Heye, T. Quantifying Radiology Resident Fatigue: Analysis of Preliminary Reports. Radiology 2021, 298, 8. [CrossRef] [PubMed]

14. Ruutiainen, A.T.; Durand, D.J.; Scanlon, M.H.; Itri, J.N. Increased Error Rates in Preliminary Reports Issued by Radiology Residents Working More Than 10 Consecutive Hours Overnight. Acad. Radiol. 2013, 20, 305-311. [CrossRef]

15. Stec, N.; Arje, D.; Moody, A.R.; Krupinski, E.A.; Tyrrell, P.N. A Systematic Review of Fatigue in Radiology: Is It a Problem? Am. J. Roentgenol. 2018, 210, 799-806. [CrossRef] [PubMed]

16. Giger, M.L. Machine Learning in Medical Imaging. J. Am. Coll. Radiol. 2018, 15, 512-520. [CrossRef]

17. Waite, S.; Kolla, S.; Jeudy, J.; Legasto, A.; Macknik, S.L.; Martinez-Conde, S.; Krupinski, E.A.; Reede, D.L. Tired in the Reading Room: The Influence of Fatigue in Radiology. J. Am. Coll. Radiol. 2017, 14, 191-197. [CrossRef]

18. Kim, H.Y.; Lee, K.; Chang, W.; Kim, Y.; Lee, S.; Oh, D.Y.; Sunwoo, L.; Lee, Y.J.; Kim, Y.H. Robustness of Deep Learning Algorithm to Varying Imaging Conditions in Detecting Low Contrast Objects in Computed Tomography Phantom Images: In Comparison to 12 Radiologists. Diagnostics 2021, 11, 410. [CrossRef] [PubMed]

19. Wu, Y.; Hatipoglu, S.; Alonso-Álvarez, D.; Gatehouse, P.; Li, B.; Gao, Y.; Firmin, D.; Keegan, J.; Yang, G. Fast and Automated Segmentation for the Three-Directional Multi-Slice Cine Myocardial Velocity Mapping. Diagnostics 2021, 11, 346. [CrossRef]

20. Weikert, T.; Winkel, D.J.; Bremerich, J.; Stieltjes, B.; Parmar, V.; Sauter, A.W.; Sommer, G. Automated Detection of Pulmonary Embolism in CT Pulmonary Angiograms Using an AI-Powered Algorithm. Eur. Radiol. 2020, 30, 6545-6553. [CrossRef]

21. Winkel, D.J.; Weikert, T.J.; Breit, H.-C.; Chabin, G.; Gibson, E.; Heye, T.J.; Comaniciu, D.; Boll, D.T. Validation of a Fully Automated Liver Segmentation Algorithm Using Multi-Scale Deep Reinforcement Learning and Comparison versus Manual Segmentation. Eur. J. Radiol. 2020, 126, 108918. [CrossRef] [PubMed]

22. Winkel, D.J.; Breit, H.-C.; Shi, B.; Boll, D.T.; Seifert, H.-H.; Wetterauer, C. Predicting Clinically Significant Prostate Cancer from Quantitative Image Features Including Compressed Sensing Radial MRI of Prostate Perfusion Using Machine Learning: Comparison with PI-RADS v2 Assessment Scores. Quant. Imaging Med. Surg. 2020, 10, 808-823. [CrossRef]

23. Zheng, Q.; Yang, L.; Zeng, B.; Li, J.; Guo, K.; Liang, Y.; Liao, G. Artificial Intelligence Performance in Detecting Tumor Metastasis from Medical Radiology Imaging: A Systematic Review and Meta-Analysis. EClinicalMedicine 2021, 31, 100669. [CrossRef]

24. Saba, L.; Biswas, M.; Kuppili, V.; Cuadrado Godia, E.; Suri, H.S.; Edla, D.R.; Omerzu, T.; Laird, J.R.; Khanna, N.N.; Mavrogeni, S.; et al. The Present and Future of Deep Learning in Radiology. Eur. J. Radiol. 2019, 114, 14-24. [CrossRef] [PubMed]

25. McBee, M.P.; Awan, O.A.; Colucci, A.T.; Ghobadi, C.W.; Kadom, N.; Kansagra, A.P.; Tridandapani, S.; Auffermann, W.F. Deep Learning in Radiology. Acad. Radiol. 2018, 25, 1472-1480. [CrossRef] [PubMed] 
26. Zhou, Y.; Xie, L.; Fishman, E.K.; Yuille, A.L. Deep Supervision for Pancreatic Cyst Segmentation in Abdominal CT Scans. In Proceedings of the Medical Image Computing and Computer Assisted Intervention-MICCAI 2017, Quebec City, QC, Canada, 11-13 September 2017; Descoteaux, M., Maier-Hein, L., Franz, A., Jannin, P., Collins, D.L., Duchesne, S., Eds.; Springer International Publishing: Cham, Germany, 2017; pp. 222-230.

27. Chu, L.C.; Park, S.; Kawamoto, S.; Wang, Y.; Zhou, Y.; Shen, W.; Zhu, Z.; Xia, Y.; Xie, L.; Liu, F.; et al. Application of Deep Learning to Pancreatic Cancer Detection: Lessons Learned From Our Initial Experience. J. Am. Coll. Radiol. 2019, 16, 1338-1342. [CrossRef] [PubMed]

28. Xie, L.; Yu, Q.; Zhou, Y.; Wang, Y.; Fishman, E.K.; Yuille, A.L. Recurrent Saliency Transformation Network for Tiny Target Segmentation in Abdominal CT Scans. IEEE Trans. Med. Imaging 2020, 39, 514-525. [CrossRef]

29. Weikert, T.; Cyriac, J.; Yang, S.; Nesic, I.; Parmar, V.; Stieltjes, B. A Practical Guide to Artificial Intelligence-Based Image Analysis in Radiology. Investig. Radiol. 2020, 55, 1-7. [CrossRef]

30. Isensee, F.; Jäger, P.F.; Kohl, S.A.A.; Petersen, J.; Maier-Hein, K.H. Automated Design of Deep Learning Methods for Biomedical Image Segmentation. Nat. Methods 2020, 18, 203-211. [CrossRef]

31. NORA-Medical Imaging Platform. Available online: Https://Www.Nora-Imaging.Com/ (accessed on 23 March 2021).

32. Vilas-Boas, F.; Macedo, G. Management Guidelines for Pancreatic Cystic Lesions: Should We Adopt or Adapt the Current Roadmaps? J. Gastrointestin. Liver Dis. 2019, 28, 495-501. [CrossRef]

33. Stutchfield, B.M.; Nayar, M.; Penman, I.D. Pancreatic Cystic Lesions: Risk Stratification and Management Based on Recent Guidelines. Frontline Gastroenterol. 2019, 10, 182-187. [CrossRef]

34. Bai, H.X.; Wang, R.; Xiong, Z.; Hsieh, B.; Chang, K.; Halsey, K.; Tran, T.M.L.; Choi, J.W.; Wang, D.-C.; Shi, L.-B.; et al. Artificial Intelligence Augmentation of Radiologist Performance in Distinguishing COVID-19 from Pneumonia of Other Origin at Chest CT. Radiology 2020, 296, E156-E165. [CrossRef]

35. Summers, R.M. Progress in Fully Automated Abdominal CT Interpretation. AJR Am. J. Roentgenol. 2016, 207, 67-79. [CrossRef] [PubMed]

36. Pandey, P.; Pandey, A.; Luo, Y.; Aliyari Ghasabeh, M.; Khoshpouri, P.; Ameli, S.; O’Broin-Lennon, A.M.; Canto, M.; Hruban, R.H.; Goggins, M.S.; et al. Follow-up of Incidentally Detected Pancreatic Cystic Neoplasms: Do Baseline MRI and CT Features Predict Cyst Growth? Radiology 2019, 292, 647-654. [CrossRef]

37. Tanaka, M.; Fernández-del Castillo, C.; Kamisawa, T.; Jang, J.Y.; Levy, P.; Ohtsuka, T.; Salvia, R.; Shimizu, Y.; Tada, M.; Wolfgang, C.L. Revisions of International Consensus Fukuoka Guidelines for the Management of IPMN of the Pancreas. Pancreatology 2017, 17, 738-753. [CrossRef]

38. Hasan, A.; Visrodia, K.; Farrell, J.J.; Gonda, T.A. Overview and Comparison of Guidelines for Management of Pancreatic Cystic Neoplasms. World J. Gastroenterol. 2019, 25, 4405-4413. [CrossRef]

39. Tanaka, M. Clinical Management and Surgical Decision-Making of IPMN of the Pancreas. In Pancreatic Cancer; Su, G.H., Ed.; Springer: New York, NY, USA, 2019; Volume 1882, pp. 9-22, ISBN 978-1-4939-8878-5.

40. van Huijgevoort, N.C.M.; del Chiaro, M.; Wolfgang, C.L.; van Hooft, J.E.; Besselink, M.G. Diagnosis and Management of Pancreatic Cystic Neoplasms: Current Evidence and Guidelines. Nat. Rev. Gastroenterol. Hepatol. 2019, 16, 676-689. [CrossRef]

41. Lee, J.E.; Choi, S.-Y.; Min, J.H.; Yi, B.H.; Lee, M.H.; Kim, S.S.; Hwang, J.A.; Kim, J.H. Determining Malignant Potential of Intraductal Papillary Mucinous Neoplasm of the Pancreas: CT versus MRI by Using Revised 2017 International Consensus Guidelines. Radiology 2019, 293, 134-143. [CrossRef]

42. Tobaly, D.; Santinha, J.; Sartoris, R.; Burgio, M.D.; Matos, C.; Cros, J.; Couvelard, A.; Rebours, V.; Sauvanet, A.; Ronot, M.; et al. CT-Based Radiomics Analysis to Predict Malignancy in Patients with Intraductal Papillary Mucinous Neoplasm (IPMN) of the Pancreas. Cancers 2020, 12, 3089. [CrossRef]

43. Sun, Y.; Yang, S.; Qi, E.; Liu, F.; Zhou, F.; Lu, Y.; Liang, P.; Ye, H.; Yu, X. Comparative Diagnostic Evaluation with ContrastEnhanced Ultrasound, Computed Tomography and Magnetic Resonance Imaging in Patients with Pancreatic Cystic Neoplasms. Cancer Manag. Res. 2020, 12, 2889-2898. [CrossRef]

44. Fábrega-Foster, K.; Kamel, I.R.; Horowitz, J.M.; Arif-Tiwari, H.; Bashir, M.R.; Chernyak, V.; Goldstein, A.; Grajo, J.R.; Hindman, N.M.; Kamaya, A.; et al. ACR Appropriateness Criteria®Pancreatic Cyst. J. Am. Coll. Radiol. 2020, 17, S198-S206. [CrossRef] [PubMed] 